\title{
HOME MANAGEMENT OF DIARRHEA AMONG UNDERFIVES IN A RURAL COMMUNITY IN KENYA: HOUSEHOLD PERCEPTIONS AND PRACTICES
}

\author{
Doreen M. Othero ${ }^{1}$, Alloys S.S Orago ${ }^{2}$, Ted Groenewegen ${ }^{3}$, Dan O. Kaseje ${ }^{4}$ and Otengah P.A ${ }^{5}$
}

\begin{abstract}
Introduction: Diarrheal disease is a major cause of morbidity and mortality among under-fives especially in rural and peri-urban communities in developing countries. Home management of diarrhea is one of the key household practices targeted for enhancement in the Community Integrated Management of Childhood IIIness (C-IMCI) strategy.

Objective: The aim of this study was to determine the perceptions of mothers/caregivers regarding the causes of diarrhea among under-fives and how it was managed in the home before seeking help from Community Health Workers or health facilities.

Design: A household longitudinal study was conducted in Nyando district, Kenya in 2004-2006 adopting both qualitative and quantitative approaches.

Subjects: A total of 927 mothers/caregivers of under-fives participated in the study.

Main outcome measures: Perceived causes of childhood diarrhoea, action taken during diarrhea, fluid intake, recognition of signs of dehydration, feeding during convalescence, adherence to treatment and advice.

Results: Majority of the respondents $807(87.1 \%)$ reported that their children had suffered from diarrhea within the last 2 weeks before commencement of the study. Diarrhea was found to contribute to $48 \%$ of child mortality in the study area. Perceived causes of diarrhea were: unclean water 524(55.6\%), contaminated food 508(54.9\%), bad eye $464(50.0 \%)$, false teeth $423(45.6 \%)$ and breast milk 331(35.8\%). More than $70 \%$ of mothers decreased fluid intake during diarrhea episodes. The mothers perceived wheat flour, rice water and selected herbs as anti-diarrheal agents. During illness, $239(27.8 \%)$ of the children were reported not to have drunk any fluids at all, 487(52.5\%) drunk much less and only $93(10.0 \%)$ were reported to have drunk more than usual. A significant $831(89.6 \%)$ withheld milk including breast milk with the notion that it enhanced diarrhea.

Conclusion: Based on these findings, there is need to develop and implement interactive communication strategies for the health workers and mothers to address perceptions and misconceptions and facilitate positive change in the household practice on management of diarrhea among under-fives.
\end{abstract}

\section{Introduction}

The survival of children in developing countries depends on the family's' and community's ability to access basic needs to support life. Each year at least 3 million children under the age of five die in the developing world due to environmental-related illnesses (1). Worldwide, diarrhea claims the lives of 2 million children each year. Over $80-90 \%$ of these cases are due to environmental conditions in particular inadequate water supply and sanitation (2). Almost 42 million people in Kenya, Uganda and Tanzania do not have access to improved water supply and 13 million do not have improved sanitation facilities (3). Evidence indicates that the primary causes of many childhood illnesses in the three countries are water related (4). Among these illnesses, diarrhea remains one of the most important environmental health problem. Integrated Management of Childhood Illness [IMCI] is the main framework within which the current child health interventions are implemented in developing countries. Stronger emphasis was initially on the treatment and management of cases. However, a community component [Community IMCI] was introduced with a set of sixteen key family practices which aim at addressing child health, survival and development at household and community levels. The family practices target among others, diarrhea prevention and control through use of safe water supplies and

Contact Author: Doreen M. Othero, Maseno University, School of Public Health and Contact Author: Doreen M. Othero, Maseno University, School of Public Health and
Community Development, Private Bag, Maseno-Kenya, E-mail: doreenamo@yahoo.com or othero@Ivbcsec.org; Tel: +254 722762222, 734893307

${ }^{1}$ Maseno University, School of Public Health, ${ }^{2}$ Kenya National AIDS Control Council, ${ }^{3}$ Kenyatta University, Communication Technology Department, ${ }^{4}$ Great Lakes University of Kisumu, ${ }^{5}$ Kenyatta University, Sociology Department

sanitation facilities, food hygiene, correct management of diarrhoea at home, prompt referral of a sick child and adherence to advice and treatment. The objective of this study was therefore to explore the mothers'/caregivers' percepulons on tne causes or aıarrnoea among unaer-fives and how it is managed at home before seeking help from skilled health service providers. The study was done in Nyando District Kenya, between 2004 and 2006. The variables investigated included: perceived causes of diarrhoea, action taken during diarrhoeal illness, recognition of signs of dehydration, feeding and fluid intake during diarrhoeal illness, feeding during convalescence and follow-up and compliance to treatment.

\section{Literature Review}

Diarrhoea remains one of the leading killers of young children. Perceptions of mothers regarding causes of diarrhoea in children are a recipe to timely and proper management at home and subsequent referral for skilled care. The education of mothers on home management of diarrhoea and the proper use of ORS can significantly reduce complications related to diarrhoea. A study by EneObong et.al (5) in Nigeria revealed that majority of mothers perceived that diarrhoea was caused by teething. In most of the cases, mothers treated diarrhoea with a combination of drugs including antibiotics, anti-diarrhoeal and herbal medicine. These drugs were prescribed mainly by mothers themselves and local shopkeepers. Knowledge on the adverse effects of the drugs was found to be minimal hence underscoring the need for appropriate primary care education among mothers.Bhutta (6) indicated that a two-week course of zinc once daily significantly reduced severity and duration of diarrhoea and mortality in young children. The need for adequate instructions to the mothers on administration of the zinc tablets was underscored. A study by Ellis et.al (7) in Mali revealed that although nearly all mothers knew that Oral Rehydration Solution (ORS) could replace lost fluids, its inability to stop diarrhoea caused them to seek antibiotics from local markets, traditional medicines or anti-malarials to cure the illness. Parents often deemed ORS insufficient 
and judged that an additional treatment should be combined with ORS to cure diarrhoea.

An observational study in Somalia by Ibrahim et.al., showed that use of ORS during childhood diarrhoea at home was popular among infants compared to older children, as were children in smaller households. ORS was used mostly by non-farming, young and literate mothers. The findings suggested that the use of ORS is associated with a mother's ability to allocate time to health care and her general position in the household since mothers in-law and husbands also made decisions on the management of sick children at home.

\section{Materials and Methods}

\section{Study area and population}

Nyando District is one of the 112 districts that make Kenya and one of the 16 districts in Nyanza Province. It lies in the eastern part of a large lowland surrounding the Nyanza Gulf, much of it comprising of predominantly black cotton soils with poor drainage and perennial flooding. The top five diseases causing morbidity and mortality among under-fives in Nyando district are: malaria, acute respiratory tract infections, malnutrition, diarrhoea and measles. Lack of access to safe drinking water is a major cause of diarrhoeal diseases. The latter are prevalent despite the fact that the district has expansive water surfaces most of which is raw and unclean water. The study population comprised of mothers/caregivers of under-fives residing in the study area for a period of not less than six months. Ministry of Health staff and Community Own Resource Persons (CORPs) were also interviewed.

\section{Data collection}

To determine the mothers'/caregivers' practices on the causes and management of diarrhoea, a semistructured questionnaire was administered to 927 randomly sampled respondents in 3 out of the 5 divisions that form the district. To explore the experiences, knowledge and perceptions on management of diarrhoea, six homogenous focus group discussions (FGDs) were held with purposively selected groups of mothers/caregivers and community health workers. A total of 42 key informants (KIs) were also interviewed including 4 health workers at policy level, 12 implementing health workers, 12 community leaders and 14 community health worker group leaders. These KIs were purposively selected based on their involvement in implementation of child health programmes or position in the community. FGD and key informant thematic guides were used to collect qualitative data.

Health workers provided information on the link between the community and the health facilities, the kind of facilitation they provided to enhance referral of children with diarrhoea and their experiences about mothers'/caregivers' health seeking behaviour.

\section{Data analysis and presentation}

Quantitative data was analyzed using SPSS version 12.0 to generate frequencies and cross tabulations. The key variables investigated were: perceived causes of diarrhoea among children, danger signs in a child with diarrhoea, remedies for treating diarrhoea at home, fluid replacement for a child with diarrhoea, feeding of a child with diarrhoea, seeking skilled treatment for a child with diarrhoea, follow-up and adherence to health worker treatment and advice. Qualitative data was manually transcribed, categorized and summarized according to the study objectives. Recurrent and emerging themes were identified and organized into meaningful categories with verbatim reporting where necessary. For comprehensiveness, quantitative and qualitative data was integrated for complementary and validation purposes.

Results

\section{Age, gender and marital status of respondents}

A total of 927 mothers/caregivers responded to the questionnaire majority of them being females 896 (96.7\%) aged between 15 and 25 years. A small proportion 28 (3.0\%) were grandparents aged between 49-56 years and were basically caring for their orphaned grandchildren. Age, gender and marital status of the respondents were not significant factors ( $P=0.06,0.08$ and 0.05 respectively).

\section{Table 1: Age and marital status of respondents}

\begin{tabular}{lll}
\hline Age Distribution & Frequency & Proportion (\%) \\
\hline 15-25yrs & 608 & 65.6 \\
$26-35$ yrs & 257 & 27.8 \\
$36-45$ yrs & 57 & 6.1 \\
>45 yrs & 5 & 0.5 \\
Total & $\mathbf{9 2 7}$ & $\mathbf{1 0 0}$ \\
& & \\
Marital status & & \\
Single & 94 & 10.1 \\
Married & 753 & 81.3 \\
Divorced & 3 & 0.3 \\
Separated & 3 & 0.3 \\
Widowed & 74 & 8.0 \\
\hline Total & $\mathbf{9 2 7}$ & $\mathbf{1 0 0}$ \\
\hline
\end{tabular}

\section{Perceived causes of diarrhoea}

A large proportion 814 (87.8\%) of the respondents affirmed that their last child had suffered from diarrhoea in the last two weeks. The commonest perceived cause of diarrhoea was unclean water 539 (58.2\%) followed by bad food 490 (52.9\%) as shown in Figure 1.

\section{Recognition of signs of dehydration}

The study investigated the mothers' and caregivers' knowledge on signs of dehydration which are indicative of severe illness. These included: excessive thirst, sunken eyes and fontanel, skin pinch returning slowly and excessive drowsiness or unconsciousness. Action taken in case of the above signs was also investigated. Majority of respondents $692(76.4 \%)$ were not able to mention any of the danger signs. Only 29 (3.1\%) of the mothers new all the danger signs. Knowledge of danger signs is important because it leads to early referral of very sick children. Failure to refer such children results in major complications or death. 


\section{Action taken during diarrhoea}

Most of the mothers reported to have given their children anti-diarrhoeal drugs (45.3\%), home-made fluids (18.7\%), taken to the health facility (14.9\%), given ORS (13.4\%) and given herbal medicine $(7.7 \%)$ as shown in figure 2 below. In-depth interviews with Community Health Workers (CHWs) as key informants revealed that mothers in the study area lacked adequate knowledge on the management of diarrhoea. They further affirmed that misconceptions of the causes of diarrhoea contributed to its mismanagement.

Figure 1: Perceived causes of diarrhoea among under-fives

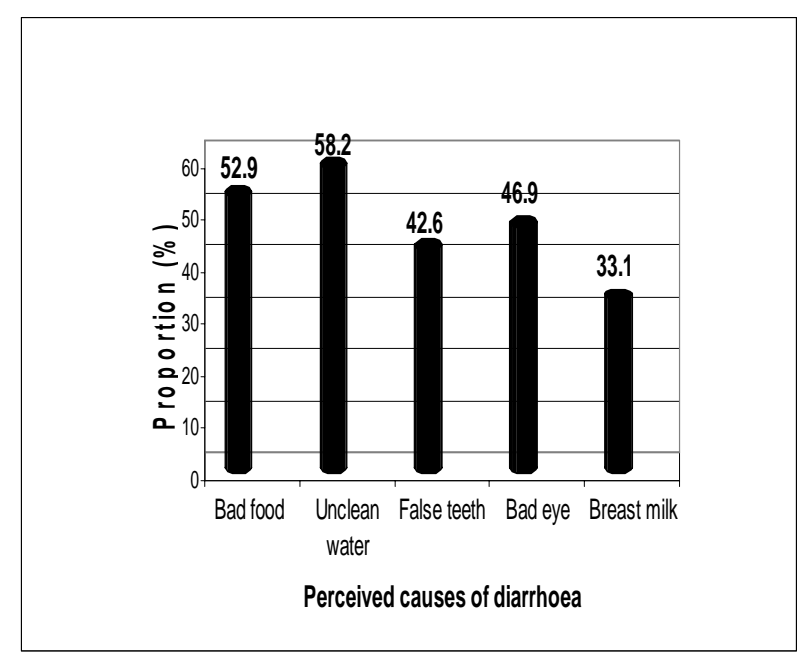

Figure 2: Action taken when child had diarrhoea

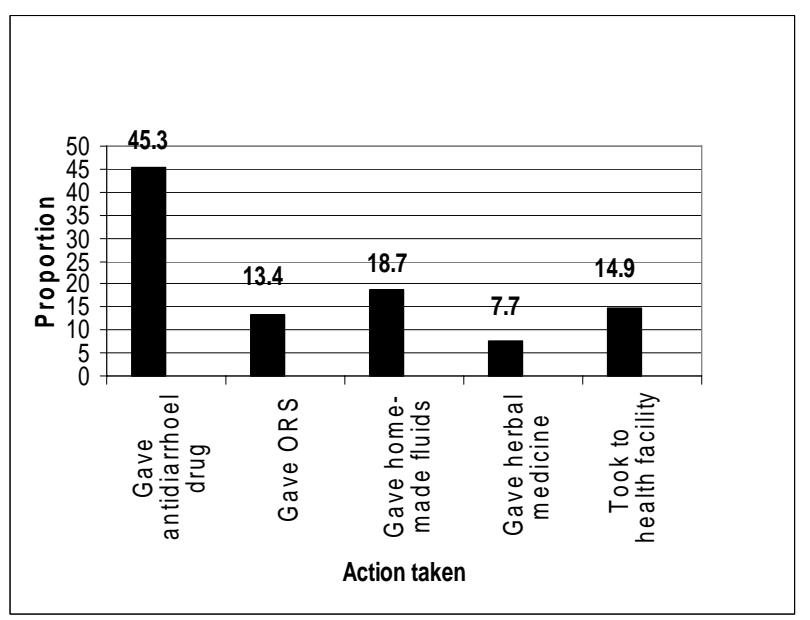

Fluid intake and feeding during diarrhoea

The study investigated the mothers'/caregivers' feeding practices during child diarrhoea. The respondents were asked whether the child was given less, same amount or more than usual to eat and/or drink during the last attack of diarrhoea. Majority of respondents 465 (50.2\%) reported that the children drunk much less, 90 (9.7\%) affirmed that the children drunk more than usual while 256 (27.6\%) reported that the child did not drink or eat anything during the last attack of diarrhoea details of which are shown in figure 3 .

Figure 3: Fluid intake during illness

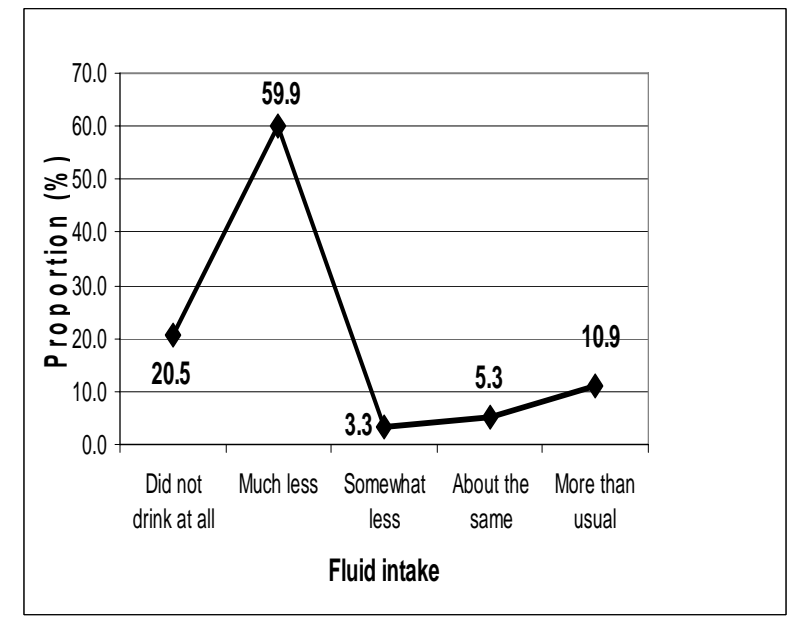

On responding to the question on whether the children ate less, the same or more than usual during illness, again responses indicated that majority of them 362(39.0\%) did not eat at all during illness, while only 64(6.9\%) ate more than usual. Those who withheld feeds and fluids felt that continued feeding increased the frequency of lose stools. One mother in a focus group discussion stated: “...the more fluids a child drinks when he/she has diarrhoea, the more frequent the lose stools and more so milk..." This misconception could result into many children suffering from dehydration and finally death.

\section{Feeding during convalescence}

On feeding during convalesce, 719 (77.6\%) of the respondents reported that the children ate the same as usual (Figure 4). Findings from focus group discussions with mother/caregivers revealed that knowledge deficit on the need to increase feeds was the main problem why some sick children were not fed adequately during or after illness. These findings depicted a dangerous trend because there was no compensation for the worn out cells and tissues as the children still fed on the usual amount they were eating before illness. This could be a recipe for malnutrition. 
Figure 4: Child feeding during convalescence

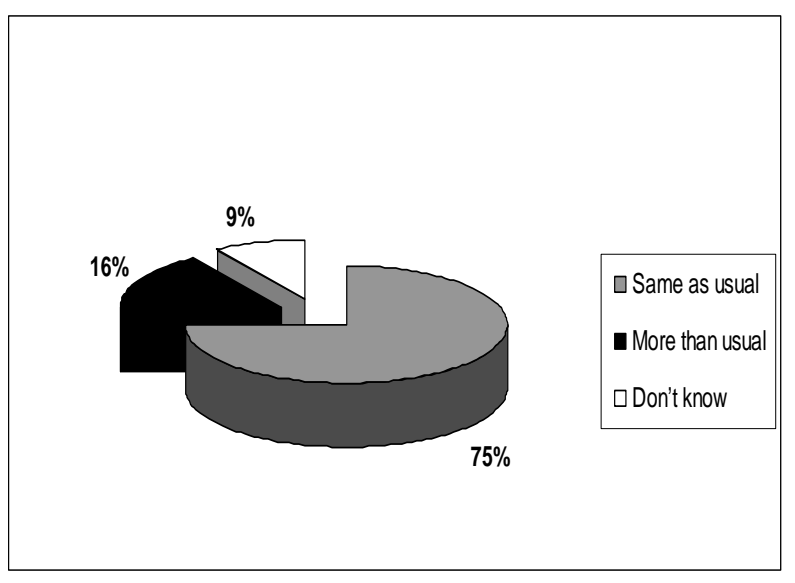

Follow-up and compliance with treatment

A significant proportion of respondents 421 (45.4\%) reported that they stopped giving the children medication on noting improvement in the condition. Most of the respondents had a misconception that too much medication in the children's bodies was not safe and they expressed that completion of the prescribed medicines would amount to overdosing the children. A small proportion 255 (27.5\%) affirmed having taken the children back to the health facilities for review following completion of medication as shown in table 2 below. Most of them did not understand why it was necessary to take the children back if the latter had improved.

Table 2: Follow-up and compliance to treatment

\begin{tabular}{llc}
\hline Follow-up and Compliance & N=927 & Proportion (\%) \\
\hline $\begin{array}{l}\text { Demonstration given on administration of } \\
\text { medicine }\end{array}$ & 305 & 32.9 \\
Medication completed & 506 & 54.6 \\
Child taken back for review & 255 & 27.5 \\
Advice given on home care & 260 & 28.1 \\
$\begin{array}{l}\text { Counselling done on prevention of } \\
\text { recurrence of infection }\end{array}$ & 180 & 19.4 \\
\hline
\end{tabular}

Multiple responses

\section{Discussion}

According to the Community Integrated Management of Childhood Illness (C-IMCI) strategy (3), caregivers at home should have adequate knowledge on the causes and treatment of diarrhoea using appropriate remedies including home-made fluids such as porridge, fresh fruit juices, milk, salt/water solution and breast milk but not herbs. Meanwhile a child is closely observed for any signs of dehydration following which urgent referral is made. This study established that most mothers gave their children anti-diarrhoeal drugs (45.3\%) and gave less fluid (50.2\%), no food (39.0\%) and argued that continued feeding made the diarrhoea worse, a practice that predisposed the child to severe dehydration. This is in line with a study in Mali by Ellis et.al (7) which revealed that nearly all parents interviewed knew that Oral Rehydration Solution (ORS) could replace lost fluids, but its inability to stop diarrhoea caused parents to seek antibiotics from local markets, traditional medicines or anti-diarrhoeal drugs to cure the illness. The notion of combining multiple treatments to ensure the greatest therapeutic benefit was prevalent, and modern medicines were often administered simultaneously with traditional therapies.

Appropriate home treatment also involves early recognition of the illness, prompt use of relevant pharmaceuticals and avoidance of ineffective and harmful treatments. Baros and Victoria (8) in their study on breast feeding and diarrhoea among Brazilian children affirmed that not all infections needed to be treated by health professionals. Uncomplicated diarrhoea could be managed successfully at home by continuing to feed the child, offering more fluids and administering Oral Rehydration Solution (ORS) correctly.

Interventions to improve home treatment of diarrhoea could reduce child deaths by up to $40 \%$. It is therefore required that a child's feeds are increased during illness in order to help the body fight the offending organisms. Due to the likely loss of appetite, the feeds should be appetizing and nutritious and given in small frequent amounts according to the child's ability to tolerate. It also recommends that during convalescence, the feeds should be increased to help the body develop new cells to replace the ones worn out during illness. This study established that most children drunk and fed less than usual when they were sick and the most common feeds were made of starchy porridge. These findings are similar to a study by Jinadu et.al (9) in Nigeria which revealed that mothers perceived raw corn starch as an antidiarrheal agent and thought that any foods with sugar including breast milk caused frequent watery stool.

This study established that a significant proportion of respondents (45.6\%) stopped giving the children medication on noting improvement in the condition. Most of the respondents observed that too much medication in the children's bodies was not safe and they felt that completion of the prescribed medicines would result in overdosing the child. A minority of the respondents (27.5\%) reported having taken the children back to the health facilities for review following completion of medication. To ensure that sick children recover quickly and completely, caregivers must adhere to the advice given by the health provider. Improving compliance to dosage and consumption of drugs is also important for reducing build up of resistance to medications. Similar findings were revealed in an evaluation survey of communication strategies for Community IMCI by USAID and DFID in 2001 as reported by WHO (2). The survey further showed that very few mothers followed advice given by the health workers and most of them tended to share the medicines with other mothers.

\section{Conclusions}

This study has demonstrated that mothers/caregivers have diverse perceptions on causes and treatment of diarrhoea some of which are harmful to the health of a child. Key among the findings is inability to recognize signs of dehydration and the use of anti-diarrhoeal drugs to stop diarrhoea. However, some reported practices such as use of home made fluids are useful hence the need for their enhancement. There is need to implement interactive 
communication strategies for mothers/caregivers and health workers at community level in order to facilitate sustainable positive change in the practice on home management of diarrhoea among under-fives. The communication strategies should recognize the key role played by culture in influencing perceptions of the mothers/caregivers.

\section{References}

1. Rukunga G, Mutethia D, Kioko T. Assessment of Environmental Interventions for Child Survival in Kenya, WELL Scoping Study 2002, WELL - WEDC, UK WHO. The Management of nutrition in major emergencies. WHO 2002 (Decision Chart), Geneva, 2002:.75

3. WHO/UNICEF. Integrated Management of Childhood Illness Information, Division of Child and Adolescent Health and Development 1999. Switzerland

4. Sharma NP et al. African Water Resources: Challenges and Opportunities for Sustainable Development. World Bank Technical Paper Africa Technical Department Series 1996, Washington, USA.

5. Ene-Obong HN, Iroegbu CU, Uwaegbute AC. Journal of Health, Population and Nutrition, 2000; 18(2):97-102.

6. Bhutta PM. Therapeutic effects of oral zinc in acute and persistent diarrhoea Bhutta PM. Therapeutic effects of oral zinc in acute and persistent diarrhoea
in children in developing countries: Pooled analysis of randomized controlled trials. American Journal of Clinical Nutrition 2000, 72(6):1516-1522).

7. Ellis AA, Winch P, Daou Z, Gilroy KE, Swedberg E. Social Science and Medicine 2007, 64(3):701-702.

8. Baros F and Victoria, G . "Breastfeeding and diarrhoea in Brazilian children". The population Council of New York, New York 2000, USA.

9. Jinadu MK, Odebiyi O, Fayewonyom BA. Tropical Medicine and Jinadu MK, Odebiyi O, Fayew
International Health 1996, 1(5):684-9.

10. Ibrahim MM, Aden AS, Omar HM, Wall S, Persson LA. Annals of Tropical Paediatrics 1994, 14(3):215-22.

11. Kenya Demographic and Health Survey. National Council for Population and Development. Ministry of Planning and National Development 1993. 